\title{
Lipschitz continuity and semiconcavity properties of the value function of a stochastic control problem
}

Rainer Buckdahn, Piermarco Cannarsa and Marc Quincampoix

\begin{abstract}
We investigate the Cauchy problem for a nonlinear parabolic partial differential equation of Hamilton-Jacobi-Bellman type and prove some regularity results, such as Lipschitz continuity and semiconcavity, for its unique viscosity solution. Our method is based on the possibility of representing such a solution as the value function of the associated stochastic optimal control problem. The main feature of our result is the fact that the solution is shown to be jointly regular in space and time without any strong ellipticity assumption on the Hamilton-Jacobi-Bellman equation.
\end{abstract}

Mathematics Subject Classification (2000). 93E20, 35D40, 35K55, 35K65.

\section{Introduction}

We consider the following partial differential equation

$$
\left\{\begin{array}{l}
-v_{t}+H\left(t, x, v,-\frac{\partial v}{\partial x},-\frac{\partial^{2} v}{\partial^{2} x}\right)=0,(t, x) \in[0, T) \times \mathbb{R}^{n} \\
v(T, x)=h(x), x \in \mathbb{R}^{n}
\end{array}\right.
$$

where

$$
\begin{aligned}
& H(t, x, u, p, A) \\
& \quad=\sup _{u \in U}\left\{\frac{1}{2} \operatorname{tr}\left(A \sigma(t, x, u) \sigma^{T}(t, x, u)\right)+\langle p, b(t, x, u)\rangle-f(t, x, u)\right\}
\end{aligned}
$$

for any $(t, x, p, A) \in[0, T] \times \mathbb{R}^{n} \times \mathbb{R}^{n} \times \mathcal{L}\left(\mathbb{R}^{n}, \mathbb{R}^{n}\right)$. It is well-known that, under continuity and boundedness assumptions on $b, \sigma, f$ and $h$, equation (1.1) has a unique continuous viscosity solution of at most polynomial growth $v$ : $[0, T] \times \mathbb{R}^{n} \mapsto \mathbb{R}$ (see, for instance, $[2]$ ). The main goal of the present article is to prove the local Lipschitz continuity and semiconcavity of the solution. We recall the following: 
Definition 1.1 ([1]) Let $A \subset \mathbb{R}^{n}$ be an open set and let $u: A \rightarrow \mathbb{R}^{n}$. We say that $f$ is semiconcave (with linear modulus) in $A$ if there exists a constant $C \geq 0$ such that, for all $\lambda \in[0,1]$,

$$
\lambda u\left(x_{1}\right)+(1-\lambda) u\left(x_{0}\right)-2 u\left(\lambda x_{1}+[1-\lambda] x_{0}\right) \leq C \lambda(1-\lambda)\left|x_{1}-x_{0}\right|^{2}
$$

for all $x_{1}, x_{0} \in A$ such that the segment $\left[x_{0}, x_{1}\right]$ is contained in $A$. Any constant $C$ satisfying the above inequality is called a semiconcavity constant for $u$ in $A$.

In the literature most of the regularity results for problem (1.1) are concerned with uniformly elliptic or parabolic equations [5,7]. For such equations solutions are expected to be smooth, even without imposing the structural assumption (1.2). In this paper, on the contrary, attention will be focussed on degenerate parabolic equations, including the fully degenerate case of $\sigma=0$ where our problem reduces to a first order equation. Therefore, one cannot expect solutions to be smooth and, as is well-known, semiconcavity is the maximal regularity that can be obtained, see. e.g., [1]. Moreover, due to the presence of a possibly degenerate diffusion in the stochastic differential equation associated with (1.1), such a regularity fails to hold at time $T$, as we shall explain later on (see Example 3.1).

The key idea of our approach is to interpret the solution of the PDE as the value function of a suitable stochastic control problem (see e.g. $[3,8]$ ), and then use fine properties of the associated control system to derive Lipschitz continuity and semiconcavity. Moreover, we obtain Lispschitz and semiconcavity constants for the solution that are independent of the specific stochastic control problem that was chosen to represent the solution of (1.1).

The main novelty of this article lies in the fact that the solution is shown to possess the same regularity with respect to both variables $x$ and $t$ (for $t \neq T$ ). Indeed, for our problem, the Lipschitz continuity or semiconcavity of the solution just with respect to space variables has been obtained:

(a) in [3] and [8], using the representation of the solution of (1.1) as the value function of the associated stochastic control problem, and

(b) in [4], by comparison arguments for viscosity solutions of Hamilton-Jacobi equations.

On the other hand, the above results only ensure space regularity, as we have already mentioned. In this paper, on the contrary, we are interested in joint space-time regularity which is an essentially new property in the present context because, in the stochastic case, time and space variables often play a different role. Indeed, although our starting point is the same representation formula for the solution of (1.1) as in (a), then we need to introduce a suitable change of time in the underlying Brownian motion and derive nontrivial estimates for the resulting stochastic integrals in order to prove our result.

Let us now explain how our paper is organized. In Sect. 2, we give our assumptions and describe the optimal stochastic control problem associated to (1.1). Section 3 is devoted to the study of the Lipschitz regularity of the solution while Sect. 4 investigates semiconcavity. 


\section{Stochastic optimal control problem}

Let $T>0$ be a fixed time horizon, let $U$ be a metric space - the control spaceand let $(\Omega, \mathcal{F}, \mathbb{P})$ be a complete probability space. For any $(s, y) \in[0, T) \times \mathbb{R}^{n}$, consider the state equation

$$
\left\{\begin{array}{l}
d x(t)=b(t, x(t), u(t)) d t+\sigma(t, x(t), u(t)) d W(t), \quad t \in[s, T], \\
x(s)=y
\end{array}\right.
$$

governed by an $m$-dimensional Brownian motion $W$ and a control process $u$.

For any $s \in[0, T)$, we define $\mathcal{U}^{w}[s, T]$ to be the set of all pairs $\nu=$ $(W(\cdot), u(\cdot))$ satisfying the following:

(i) $\{W(t)\}_{t \geq s}$ is an $m$-dimensional standard Brownian motion on $(\Omega, \mathcal{F}, \mathbb{P})$ over $[s, \bar{T}]$ (with $W(s)=0$ almost surely) for which we denote by $\mathcal{F}_{t}^{s}=$ $\sigma\{W(r): s \leq r \leq t\}$ the associated filtration, augmented by all the $\mathbb{P}$-null sets of $\mathcal{F}$.

(ii) $u \in L_{\left\{\mathcal{F}_{t}^{s}\right\}_{t \geq s}}^{0}(0, T ; U)$ is an $\left\{\mathcal{F}_{t}^{s}\right\}_{t \geq s}-\operatorname{adapted}$ process on $(\Omega, \mathcal{F}, \mathbb{P})$.

Sometimes, we simply write $u(\cdot) \in \mathcal{U}^{w}[s, T]$ instead of $(W(\cdot), u(\cdot)) \in \mathcal{U}^{w}[s, T]$.

Under standard assumptions, that will be explicitly recalled below, for any $y \in \mathbb{R}^{n}$ Eq. (2.3) admits a unique solution $x(\cdot)$. Then, for any $(s, y) \in$ $[0, T] \times \mathbb{R}^{n}$ and $u \in \mathcal{U}^{w}[s, T]$ one can compute the cost

$$
J(s, y ; u)=\mathbb{E}\left\{\int_{s}^{T} f(t, x(t), u(t)) d t+h(x(T))\right\}
$$

and the corresponding value function

$$
V(s, y)=\inf _{u(\cdot) \in \mathcal{U}^{w}[s, T]} J(s, y ; u) \quad \forall(s, y) \in[0, T] \times \mathbb{R}^{n} .
$$

We underline that the mathematical expectation $\mathbb{E}$ in (2.4) is taken with respect to probability measure $\mathbb{P}$.

Let us now introduce some assumptions.

(S1) $U$ is a complete separable metric space.

(S2) Maps b: $[0, T] \times \mathbb{R}^{n} \times U \rightarrow \mathbb{R}^{n}, \sigma:[0, T] \times \mathbb{R}^{n} \times U \rightarrow \mathbb{R}^{n \times m}, f:$ $[0, T] \times \mathbb{R}^{n} \times U \rightarrow \mathbb{R}$ and $h: \mathbb{R}^{n} \rightarrow \mathbb{R}$ are uniformly continuous and there exists a positive constant $L$ such that the function

$$
\phi(t, x, u)=(b(t, x, u), \sigma(t, x, u), f(t, x, u), h(x))
$$

satisfies

$$
\begin{aligned}
|\phi(t, x, u)-\phi(t, \hat{x}, u)| & \leq L|x-\hat{x}| \quad \forall t \in[0, T], x, \hat{x} \in \mathbb{R}^{n}, u \in U, \\
|\phi(t, x, u)| & \leq L \quad \forall(t, x) \in[0, T] \times \mathbb{R}^{n}, u \in U .
\end{aligned}
$$

Under assumptions $(S 1)$ and $(S 2)$, for any $(s, y) \in[0, T] \times \mathbb{R}^{n}$ and $u(\cdot) \in$ $\mathcal{U}^{w}[s, T],(2.3)$ admits a unique solution $x(\cdot)$ and the cost functional (2.4) is well-defined. We also recall that $V$ is the unique continuous viscosity solution of (1.1) with at most polynomial growth (see $[2,3,8])$. 
Remark 2.1. In fact, (2.3) is well-posed even when functions $b, \sigma$ and $f$ have linear growth with respect to $x$-instead of being bounded as assumed in (S2). The results of our paper remain valid under linear growth conditions but, for simplicity, here we prefer to restrict the analysis to the bounded case.

\section{Lipschitz continuity}

The fact that, under assumption $(S 1)$ and $(S 2)$, the solution to (1.1) ought to be jointly Lipschitz continuous in $(t, x)$ is somewhat expected in the literature, see, e.g., $[4,6]$. On the other hand, some care is needed to give a precise Lipschitz regularity result for $V$, as our next example shows.

Example 3.1. We consider the following one dimensional example $m=n=1$ without control where $b=0, \sigma=1, f=0$ are constant and $h(x)=|x|$, for any $x \in \mathbb{R}$. Then it can be easily checked that $V(s, 0)=\sqrt{T-s}$ and so $V$ fails to be Lipschitz with respect to $s$ at $s=T$.

The theorem below completes the analysis of the Lipschitz regularity of $V$.

Theorem 3.2. Under assumptions $(S 1)$ and $(S 2)$ the value function $V$ is Lipschitz continuous in $[0, T-\delta) \times \mathbb{R}^{n}, \forall \delta>0$.

We give a complete proof of the above theorem using a technique that is similar - yet simpler - to the one that will be needed later for semiconcavity.

Proof. We remark that K, during the proof, denotes a generic constant, that may differ at different places.

Let us fix $\delta>0$, and let $\left(s_{1}, y_{1}\right)$ and $\left(s_{0}, y_{0}\right) \in[0, T) \times \mathbb{R}^{n}$ be such that

$$
\min \left\{T-s_{1}, T-s_{0}\right\}>\delta .
$$

We have to show that

$$
V\left(s_{1}, y_{1}\right)-V\left(s_{0}, y_{0}\right) \leq C\left(\left|s_{1}-s_{0}\right|+\left|y_{1}-y_{0}\right|\right)
$$

for some constant $C \geq 0$. For any $\epsilon>0$, there exists $u_{0}(\cdot) \in \mathcal{U}^{w}\left[s_{0}, T\right]$ such that $J\left(s_{0}, y_{0} ; u_{0}\right)<V\left(s_{0}, y_{0}\right)+\epsilon$. Let $x_{0}(\cdot)$ be the solution of

$$
\left\{\begin{array}{l}
d x_{0}(t)=b\left(t, x_{0}(t), u_{0}(t)\right) d t+\sigma\left(t, x_{0}(t), u_{0}(t)\right) d W(t), t \in\left[s_{0}, T\right], \\
x_{0}\left(s_{0}\right)=y_{0} .
\end{array}\right.
$$

Now, let us consider the change of time

$$
\tau:\left[s_{1}, T\right] \rightarrow\left[s_{0}, T\right], \quad \tau(t)=\frac{T\left(s_{0}-s_{1}\right)+\left(T-s_{0}\right) t}{T-s_{1}}
$$

Observe that $\dot{\tau}(t)$ is constant, more precisely

$$
\dot{\tau}(t)=\frac{T-s_{0}}{T-s_{1}} .
$$


Define $u_{1}(t)=u_{0}(\tau(t))$, and denote by $x_{1}(t)$ the solution of

$$
\left\{\begin{array}{l}
d x_{1}(t)=b\left(t, x_{1}(t), u_{1}(t)\right) d t+\sigma\left(t, x_{1}(t), u_{1}(t)\right) d W_{1}(t), t \in\left[s_{1}, T\right], \\
x_{1}\left(s_{1}\right)=y_{1},
\end{array}\right.
$$

where $W_{1}(t)=\sqrt{1 / \dot{\tau}} W(\tau(t))$. Obviously, $\left(\Omega, \mathcal{F}, \mathbb{P}, W_{1}(\cdot), u_{1}(\cdot)\right) \in \mathcal{U}^{w}\left[s_{1}, T\right]$. Thus,

$$
\begin{aligned}
x_{1}(t) & =y_{1}+\int_{s_{1}}^{t} b\left(s, x_{1}(s), u_{1}(s)\right) d s+\int_{s_{1}}^{t} \sigma\left(s, x_{1}(s), u_{1}(s)\right) d W_{1}(s) \\
& =y_{1}+\int_{s_{1}}^{t} b\left(s, x_{1}(s), u_{0}(\tau(s))\right) d s+\int_{s_{1}}^{t} \sqrt{1 / \dot{\tau}} \sigma\left(s, x_{1}(s), u_{0}(\tau(s))\right) d W(\tau(s))
\end{aligned}
$$

Next, the very definition of $V$ yields

$$
\begin{aligned}
& V\left(s_{1}, y_{1}\right)-V\left(s_{0}, y_{0}\right)-\epsilon \leq J\left(s_{1}, y_{1}, u_{1}\right)-J\left(s_{0}, y_{0}, u_{0}\right) \\
& \quad=\mathbb{E}\left\{\int_{s_{1}}^{T} f\left(t, x_{1}(t), u_{1}(t)\right) d t-\int_{s_{0}}^{T} f\left(t, x_{0}(t), u_{0}(t)\right) d t+h\left(x_{1}(T)\right)-h\left(x_{0}(T)\right)\right\}
\end{aligned}
$$

By the change of variable $r=\tau(t)$ in the first integral above we obtain, setting $\tilde{x}_{1}(r)=x_{1}\left(\tau^{-1}(r)\right)$,

$$
\begin{aligned}
& V\left(s_{1}, y_{1}\right)-V\left(s_{0}, y_{0}\right)-\epsilon \\
& \leq \mathbb{E}\left\{\int_{s_{0}}^{T}\left[\frac{1}{\dot{\tau}} f\left(\tau^{-1}(r), \tilde{x}_{1}(r), u_{0}(r)\right)-f\left(r, x_{0}(r), u_{0}(r)\right)\right] d r\right\} \\
& \quad+\mathbb{E}\left\{h\left(x_{1}(T)\right)-h\left(x_{0}(T)\right)\right\}
\end{aligned}
$$

Recalling that $f(t, x, u)$ and $h(x)$ are bounded and Lipschitz, we have

$$
\begin{aligned}
& V\left(s_{1}, y_{1}\right)-V\left(s_{0}, y_{0}\right)-\epsilon \\
& \leq \mathbb{E}\left\{\int_{s_{0}}^{T}\left[\frac{1}{\dot{\tau}} f\left(\tau^{-1}(r), \tilde{x}_{1}(r), u_{0}(r)\right)-f\left(\tau^{-1}(r), \tilde{x}_{1}(r), u_{0}(r)\right)\right] d r\right\} \\
&+\mathbb{E}\left\{\int_{s_{0}}^{T}\left[f\left(\tau^{-1}(r), \tilde{x}_{1}(r), u_{0}(r)\right)-f\left(r, x_{0}(r), u_{0}(r)\right)\right] d r\right\} \\
&+\mathbb{E}\left\{h\left(x_{1}(T)\right)-h\left(x_{0}(T)\right)\right\} \\
& \leq K \mathbb{E}\left\{\int_{s_{0}}^{T}\left(\left|1-\frac{1}{\dot{\tau}}\right|+\left|\tau^{-1}(r)-r\right|+\left|\tilde{x}_{1}(r)-x_{0}(r)\right|\right) d r\right\} \\
&+K \mathbb{E}\left\{\left|x_{1}(T)-x_{0}(T)\right|\right\}
\end{aligned}
$$

So, the conclusion (3.7) will follow from the estimates

$$
\begin{aligned}
\left|1-\frac{1}{\dot{\tau}}\right| & \leq K\left|s_{1}-s_{0}\right| \\
\left|\tau^{-1}(r)-r\right| d r & \leq K\left|s_{1}-s_{0}\right| \\
\mathbb{E}\left\{\int_{s_{0}}^{T}\left|\tilde{x}_{1}(r)-x_{0}(r)\right| d r\right\} & \leq K\left(\left|y_{1}-y_{0}\right|+\left|s_{1}-s_{0}\right|\right)
\end{aligned}
$$


and

$$
\mathbb{E}\left\{\left|x_{1}(T)-x_{0}(T)\right|\right\} \leq K\left(\left|y_{1}-y_{0}\right|+\left|s_{1}-s_{0}\right|\right) .
$$

Proof of (3.12). In view of (3.10) and (3.6) we have

$$
\left|1-\frac{1}{\dot{\tau}}\right|=\left|1-\frac{T-s_{1}}{T-s_{0}}\right|=\left|\frac{s_{1}-s_{0}}{T-s_{0}}\right| \leq \frac{1}{\delta}\left|s_{1}-s_{0}\right| \leq K\left|s_{1}-s_{0}\right|,
$$

which in turn yields (3.12).

Proof of (3.13). By the definition of $\tau(t)$ and assumption (3.6) we obtain $\left|\tau^{-1}(r)-r\right|=\left|\frac{\left(T-s_{1}\right) r-\left(s_{0}-s_{1}\right) T}{\left(T-s_{0}\right)}-r\right|=\left|\frac{\left(s_{1}-s_{0}\right)(T-r)}{\left(T-s_{0}\right)}\right| \leq K\left|s_{1}-s_{0}\right|$

Proof of (3.14). Recalling the definition of $\tilde{x}_{1}(r)$ we conclude that

$$
\begin{aligned}
& \mathbb{E}\left\{\left|\tilde{x}_{1}(r)-x_{0}(r)\right|^{2}\right\}=\mathbb{E}\left\{\left|x_{1}\left(\tau^{-1}(r)\right)-x_{0}(r)\right|^{2}\right\} \\
& \leq \mathbb{E}\left\{\mid y_{1}-y_{0}+\int_{s_{1}}^{\tau^{-1}(r)} b\left(s, x_{1}(s), u_{0}(\tau(s))\right) d s-\int_{s_{0}}^{r} b\left(s, x_{0}(s), u_{0}(s)\right) d s\right. \\
& \quad+\int_{s_{1}}^{\tau^{-1}(r)} \sqrt{1 / \dot{\tau}} \sigma\left(s, x_{1}(s), u_{0}(\tau(s))\right) d W(\tau(s)) \\
&\left.\quad-\left.\int_{s_{0}}^{r} \sigma\left(s, x_{0}(s), u_{0}(s)\right) d W(s)\right|^{2}\right\} \\
&=\mathbb{E}\left\{\mid y_{1}-y_{0}+\int_{s_{0}}^{r}\left[\dot{\tau}^{-1} b\left(\tau^{-1}(\rho), \tilde{x}_{1}(\rho), u_{0}(\rho)\right)-b\left(\rho, x_{0}(\rho), u_{0}(\rho)\right)\right] d \rho\right. \\
&\left.\quad+\left.\int_{s_{0}}^{r}\left[\dot{\tau}^{-1 / 2} \sigma\left(\tau^{-1}(\rho), \tilde{x}_{1}(\rho), u_{0}(\rho)\right)-\sigma\left(\rho, x_{0}(\rho), u_{0}(\rho)\right)\right] d W(\rho)\right|^{2}\right\} \\
& \leq K\left(\left|y_{1}-y_{0}\right|^{2}+K \mathbb{E}\left\{\left[\int _ { s _ { 0 } } ^ { r } \left(\left|\dot{\tau}^{-1}-1\right|\left|b\left(\tau^{-1}(\rho), \tilde{x}_{1}(\rho), u_{0}(\rho)\right)\right|\right.\right.\right.\right. \\
&\left.\left.\left.+\left|b\left(\tau^{-1}(\rho), \tilde{x}_{1}(\rho), u_{0}(\rho)\right)-b\left(\rho, x_{0}(\rho), u_{0}(\rho)\right)\right|\right) d \rho\right]^{2}\right\} \\
&+K \mathbb{E}_{s_{0}}^{r}\left|\dot{\tau}^{-1 / 2}-1\right|^{2}\left|\sigma\left(\tau^{-1}(\rho), \tilde{x}_{1}(\rho), u_{0}(\rho)\right)\right|^{2} d \rho \\
&+K \mathbb{E}_{s_{0}}^{r}\left|\sigma\left(\tau^{-1}(\rho), \tilde{x}_{1}(\rho), u_{0}(\rho)\right)-\sigma\left(\rho, x_{0}(\rho), u_{0}(\rho)\right)\right|^{2} d \rho
\end{aligned}
$$

Since $b(t, x, u)$ and $\sigma(t, x, u)$ are bounded Lipschitz functions,

$$
\begin{aligned}
& \mathbb{E}\left\{\left|\tilde{x}_{1}(r)-x_{0}(r)\right|^{2}\right\} \\
& \quad \leq K\left|y_{1}-y_{0}\right|^{2}+K \mathbb{E}\left\{\int_{s_{0}}^{r}\left[\left|s_{1}-s_{0}\right|^{2}+\left|\tilde{x}_{1}(\rho)-\tilde{x}_{0}(\rho)\right|^{2}\right] d \rho\right\} \\
& \quad \leq K\left(\left|y_{1}-y_{0}\right|^{2}+\left|s_{1}-s_{0}\right|^{2}\right)+K \int_{s_{0}}^{r} \mathbb{E}\left\{\left|\tilde{x}_{1}(\rho)-\tilde{x}_{0}(\rho)\right|^{2}\right\} d \rho
\end{aligned}
$$


Finally, Gronwall's Lemma yields

$$
\mathbb{E}\left\{\left|\tilde{x}_{1}(r)-x_{0}(r)\right|^{2}\right\} \leq K\left(\left|y_{1}-y_{0}\right|^{2}+\left|s_{1}-s_{0}\right|^{2}\right) .
$$

From the latter estimate, (3.14) easily follows.

proof[Proof of (3.15)] Owing to (3.16),

$$
\mathbb{E}\left\{\left|x_{1}(T)-x_{0}(T)\right|\right\}=\mathbb{E}\left\{\left|\tilde{x}_{1}(T)-x_{0}(T)\right|\right\} \leq K\left(\left|y_{1}-y_{0}\right|+\left|s_{1}-s_{0}\right|\right) .
$$

This yields (3.15) and completes the proof.

\section{Semiconcavity}

Theorem 4.1. In addition to $(S 1)$ and $(S 2)$, assume:

(S3)(i) $\quad h$ is semiconcave in $\mathbb{R}^{n}$ and $f(\cdot, \cdot, u)$ is semiconcave in $[0, T] \times \mathbb{R}^{n}$ uniformly in $u \in U$;

(S3)(ii) $b$ and $\sigma$ are continuously differentiable in $(t, x)$ with derivatives $b_{t, x}$ and $\sigma_{t, x}$ satisfying, for some constant $\ell \geq 0$,

$$
\begin{aligned}
& \left|b_{t, x}(t, x, u)-b_{t, x}(s, y, u)\right|+\left|\sigma_{t, x}(t, x, u)-\sigma_{t, x}(s, y, u)\right| \\
& \quad \leq \ell(|x-y|+|t-s|)
\end{aligned}
$$

for all $t, s \in[0, T]$, all $x, y \in \mathbb{R}^{n}$, and all $u \in U$.

Then the value function $V$ is semiconcave in $[0, T-\delta) \times \mathbb{R}^{n}$ for every $\delta>0$

Proof. Once again $K$ will denote a generic constant, that may differ from line to line. Let $\delta>0$ be fixed and let $\left(s_{1}, y_{1}\right),\left(s_{0}, y_{0}\right) \in[0, T) \times \mathbb{R}^{n}$ be such that

$$
\min \left\{T-s_{1}, T-s_{0}\right\}>\delta .
$$

We only have to show that, for some constant $C \geq 0$ and all $\lambda \in[0,1]$,

$$
\begin{gathered}
\lambda V\left(s_{1}, y_{1}\right)+(1-\lambda) V\left(s_{0}, y_{0}\right)-V\left(s_{\lambda}, y_{\lambda}\right) \\
\leq C \lambda(1-\lambda)\left(\left|s_{1}-s_{0}\right|^{2}+\left|y_{1}-y_{0}\right|^{2}\right)
\end{gathered}
$$

where $\left(s_{\lambda}, y_{\lambda}\right) \doteq \lambda\left(s_{1}, y_{1}\right)+(1-\lambda)\left(s_{0}, y_{0}\right)$. Let $\lambda \in[0,1]$ be fixed.

For any $\epsilon>0$, there exists a control $u_{\lambda, \epsilon}(\cdot)=u_{\lambda}(\cdot) \in \mathcal{U}^{w}\left[s_{0}, T\right]$ such that

$$
J\left(s_{\lambda}, y_{\lambda}, u_{\lambda}\right)<V\left(s_{\lambda}, y_{\lambda}\right)+\epsilon .
$$

Let $x_{\lambda}(\cdot)$ be the solution of the Cauchy problem

$$
\left\{\begin{array}{l}
d x_{\lambda}(t)=b\left(t, x_{\lambda}(t), u_{\lambda}(t)\right) d t+\sigma\left(t, x_{\lambda}(t), u_{\lambda}(t)\right) d W(t), t \in\left[s_{\lambda}, T\right], \\
x_{\lambda}\left(s_{\lambda}\right)=y_{\lambda} .
\end{array}\right.
$$

Now, for $i=0,1$ consider the time changes

$$
\tau_{i}:\left[s_{i}, T\right] \rightarrow\left[s_{\lambda}, T\right], \tau_{i}(t)=\frac{T\left(s_{\lambda}-s_{i}\right)+\left(T-s_{\lambda}\right) t}{T-s_{i}}
$$

and note that $\dot{\tau}_{i}(t)$ is constant, with $\dot{\tau}_{i}(t)=\left(T-s_{\lambda}\right) /\left(T-s_{i}\right), t \in\left[s_{i}, T\right]$. Finally, define $u_{i}(t)=u_{\lambda}\left(\tau_{i}(t)\right)$ and let $x_{i}(t)$ be the solution of the problem

$$
\left\{\begin{array}{l}
d x_{i}(t)=b\left(t, x_{i}(t), u_{i}(t)\right) d t+\sigma\left(t, x_{i}(t), u_{i}(t)\right) d W_{i}(t), t \in\left[s_{i}, T\right], \\
x_{i}\left(s_{i}\right)=y_{i},
\end{array}\right.
$$

where $W_{i}(t)=\sqrt{1 / \dot{\tau}_{i}} W\left(\tau_{i}(t)\right)$. 
By the very definition of $V$, we then have:

$$
\begin{aligned}
\lambda V & \left(s_{1}, y_{1}\right)+(1-\lambda) V\left(s_{0}, y_{0}\right)-V\left(s_{\lambda}, y_{\lambda}\right)-\epsilon \\
\leq & \lambda J\left(s_{1}, y_{1}, u_{1}\right)+(1-\lambda) J\left(s_{0}, y_{0}, u_{0}\right)-J\left(s_{\lambda}, y_{\lambda}, u_{\lambda}\right) \\
= & \mathbb{E}\left\{\lambda \int_{s_{1}}^{T} f\left(t, x_{1}(t), u_{1}(t)\right) d t+(1-\lambda) \int_{s_{0}}^{T} f\left(t, x_{0}(t), u_{0}(t)\right) d t\right\} \\
& -\mathbb{E}\left\{\int_{s_{\lambda}}^{T} f\left(y, x_{\lambda}(t), u_{\lambda}(t)\right) d t\right\} \\
& +\mathbb{E}\left\{\lambda h\left(x_{1}(T)\right)+(1-\lambda) h\left(x_{0}(T)\right)-h\left(x_{\lambda}(T)\right)\right\}
\end{aligned}
$$

In the first two integrals above let us apply the change of variables $r=\tau_{i}(t)$, defined by (4.20). Then, for $\tilde{x}_{i}(r) \doteq x_{i}\left(\tau_{i}^{-1}(r)\right)(i=0,1)$, we have

$$
\begin{aligned}
\lambda V\left(s_{1}, y_{1}\right)+(1-\lambda) V\left(s_{0}, y_{0}\right)-V\left(s_{\lambda}, y_{\lambda}\right)-\epsilon \\
\leq \mathbb{E}\left\{\int _ { s _ { \lambda } } ^ { T } \left[\frac{\lambda}{\dot{\tau}_{1}} f\left(\tau_{1}^{-1}(r), \tilde{x}_{1}(r), u_{\lambda}(r)\right)+\frac{1-\lambda}{\dot{\tau}_{0}} f\left(\tau_{0}^{-1}(r), \tilde{x}_{0}(r), u_{\lambda}(r)\right)\right.\right. \\
\left.\left.\quad-f\left(r, x_{\lambda}(r), u_{\lambda}(r)\right)\right] d r\right\} \\
\quad+E\left\{\lambda h\left(x_{1}(T)\right)+(1-\lambda) h\left(x_{0}(T)\right)-h\left(\left(x_{\lambda}(T)\right)\right)\right\}
\end{aligned}
$$

Now, observe that $\tilde{\lambda}_{1} \doteq \lambda / \dot{\tau}_{1}$ and $\tilde{\lambda}_{0} \doteq(1-\lambda) / \dot{\tau}_{0}$ satisfy $\tilde{\lambda}_{1}+\tilde{\lambda}_{0}=1$, and define

$$
\left\{\begin{array}{l}
\tilde{t}(r)=\tilde{\lambda}_{1} \tau_{1}^{-1}(r)+\tilde{\lambda}_{0} \tau_{0}^{-1}(r) \\
\tilde{x}_{\lambda}(r)=\tilde{\lambda}_{1} \tilde{x}_{1}(r)+\tilde{\lambda}_{0} \tilde{x}_{0}(r) \\
\tilde{X}_{\lambda}(r)=\lambda x_{1}(r)+(1-\lambda) x_{0}(r)
\end{array}\right.
$$

By the semiconcavity and Lipschitzianity of $f$ and $h$ we obtain

$$
\begin{aligned}
\lambda V\left(s_{1}, y_{1}\right)+(1-\lambda) V\left(s_{0}, y_{0}\right)-V\left(s_{\lambda}, y_{\lambda}\right)-\epsilon & \\
\leq & \mathbb{E}\left\{\int _ { s _ { \lambda } } ^ { T } \left[\tilde{\lambda}_{1} f\left(\tau_{1}^{-1}(r), \tilde{x}_{1}(r), u_{\lambda}(r)\right)+\tilde{\lambda}_{0} f\left(\tau_{0}^{-1}(r), \tilde{x}_{0}(r), u_{\lambda}(r)\right)\right.\right. \\
& \left.\left.-f\left(\tilde{t}(r), \tilde{x}_{\lambda}(r), u_{\lambda}(r)\right)\right] d r\right\} \\
& +\mathbb{E}\left\{\int_{s_{\lambda}}^{T}\left[f\left(\tilde{t}(r), \tilde{x}_{\lambda}(r), u_{\lambda}(r)\right)-f\left(r, x_{\lambda}(r), u_{\lambda}(r)\right)\right] d r\right\} \\
& +\mathbb{E}\left\{\lambda h\left(x_{1}(T)\right)+(1-\lambda) h\left(x_{0}(T)\right)-h\left(\tilde{X}_{\lambda}(T)\right)+h\left(\tilde{X}_{\lambda}(T)\right)-h\left(x_{\lambda}(T)\right)\right\} \\
\leq & K \tilde{\lambda}_{0} \tilde{\lambda}_{1} \mathbb{E}\left\{\int_{s_{\lambda}}^{T}\left[\left|\tau_{1}^{-1}(r)-\tau_{0}^{-1}(r)\right|^{2}+\left|\tilde{x}_{1}(r)-\tilde{x}_{0}(r)\right|^{2}\right] d r\right\}
\end{aligned}
$$




$$
\begin{aligned}
& +K \mathbb{E}\left\{\int_{s_{\lambda}}^{T}\left(|\tilde{t}(r)-r|+\left|\tilde{x}_{\lambda}(r)-x_{\lambda}(r)\right|\right) d r\right\} \\
& +K \lambda(1-\lambda) \mathbb{E}\left\{\left|x_{1}(T)-x_{0}(T)\right|^{2}\right\}+K \mathbb{E}\left\{\left|\tilde{X}_{\lambda}(T)-x_{\lambda}(T)\right|\right\}
\end{aligned}
$$

Now, the technical lemmas below will provide all the estimates that are needed to complete the proof.

Lemma 4.2. The following estimates hold true:

$$
\begin{aligned}
\tilde{\lambda}_{0} \tilde{\lambda}_{1} \int_{s_{\lambda}}^{T}\left|\tau_{1}^{-1}(r)-\tau_{0}^{-1}(r)\right|^{2} d r & \leq K \lambda(1-\lambda)\left|s_{1}-s_{0}\right|^{2} \\
\tilde{\lambda}_{0} \tilde{\lambda}_{1} \mathbb{E}\left\{\int_{s_{\lambda}}^{T}\left|\tilde{x}_{1}(r)-\tilde{x}_{0}(r)\right|^{2} d r\right\} & \leq K \lambda(1-\lambda)\left(\left|s_{1}-s_{0}\right|^{2}+\left|y_{1}-y_{0}\right|^{2}\right) \\
|\tilde{t}(r)-r| & \leq K \lambda(1-\lambda)\left|s_{1}-s_{0}\right|^{2} \\
\mathbb{E}\left\{\int_{s_{\lambda}}^{T}\left|\tilde{x}_{\lambda}(r)-x_{\lambda}(r)\right|^{2} d r\right\} & \leq K \lambda(1-\lambda)\left(\left|s_{1}-s_{0}\right|^{2}+\left|y_{1}-y_{0}\right|^{2}\right)
\end{aligned}
$$

and

$$
\mathbb{E}\left\{\left|\tilde{X}_{\lambda}(T)-x_{\lambda}(T)\right|\right\} \leq K \lambda(1-\lambda)\left(\left|y_{1}-y_{0}\right|^{2}+\left|s_{1}-s_{0}\right|^{2}\right) .
$$

Proof of Lemma 4.2. We first observe that directly from the definitions of $\tau_{i}$ and $\tilde{\lambda}_{i}$ it follows that

$$
\left|\tau_{1}^{-1}(r)-\tau_{0}^{-1}(r)\right|=\left|\frac{\left(s_{1}-s_{0}\right)(T-r)}{T-s_{\lambda}}\right| \leq\left|s_{1}-s_{0}\right|
$$

and

$$
\tilde{\lambda}_{0} \tilde{\lambda}_{1}=\frac{\lambda(1-\lambda)}{\dot{\tau}_{1} \dot{\tau}_{0}} \leq \lambda(1-\lambda)
$$

That proves (4.23).

Inequality (4.25) can be checked by a straightforward computation that we omit.

Proof of (4.24). By the definition of $\tilde{x}_{i}, i=0,1$, and a change of variables we have

$$
\begin{aligned}
& \mathbb{E}\left\{\left|\tilde{x}_{1}(r)-\tilde{x}_{0}(r)\right|^{4}\right\} \\
& =\mathbb{E}\left\{\mid y_{1}-y_{0}+\int_{s_{\lambda}}^{r}\left[\dot{\tau}_{1}^{-1} b\left(\tau_{1}^{-1}(\rho), \tilde{x}_{1}(\rho), u_{\lambda}(\rho)\right)-\dot{\tau}_{0}^{-1} b\left(\tau_{0}^{-1}(\rho), \tilde{x}_{0}(\rho), u_{\lambda}(\rho)\right)\right] d \rho\right. \\
& \left.\quad+\left.\int_{s_{\lambda}}^{r}\left[\dot{\tau}_{1}^{-1 / 2} \sigma\left(\tau_{1}^{-1}(\rho), \tilde{x}_{1}(\rho), u_{\lambda}(\rho)\right)-\dot{\tau}_{0}^{-1 / 2} \sigma\left(\tau_{0}^{-1}(\rho), \tilde{x}_{0}(\rho), u_{\lambda}(\rho)\right)\right] d W(\rho)\right|^{4}\right\}
\end{aligned}
$$


Hence, owing to the Lipschitz continuity and boundedness of $b$ and $\sigma$,

$$
\begin{aligned}
& \mathbb{E}\left\{\left|\tilde{x}_{1}(r)-\tilde{x}_{0}(r)\right|^{4}\right\} \leq K\left|y_{1}-y_{0}\right|^{4} \\
& \quad+K \mathbb{E}\left\{\left[\int _ { s _ { \lambda } } ^ { r } \left(\left|\dot{\tau}_{1}^{-1}-\dot{\tau}_{0}^{-1}\right|+\left|\dot{\tau}_{0}^{-1}\right|\left|\tilde{x}_{1}(\rho)-\tilde{x}_{0}(\rho)\right|\right.\right.\right. \\
& \left.\left.\left.\quad+\left|\dot{\tau}_{0}^{-1}\right|\left|\tau_{1}^{-1}(\rho)-\tau_{0}^{-1}(\rho)\right|\right) d \rho\right]^{4}\right\} \\
& \quad+K \mathbb{E}\left\{\left(\int _ { s _ { \lambda } } ^ { r } \left[\left|\dot{\tau}_{1}^{-1 / 2}-\dot{\tau}_{0}^{-1 / 2}\right|+\left|\dot{\tau}_{0}^{-1 / 2}\right|\left|\tilde{x}_{1}(\rho)-\tilde{x}_{0}(\rho)\right|\right.\right.\right. \\
& \left.\left.\left.\quad+\left|\dot{\tau}_{0}^{-1 / 2}\right|\left|\tau_{1}^{-1}(\rho)-\tau_{0}^{-1}(\rho)\right|\right]^{2} d \rho\right)^{2}\right\}
\end{aligned}
$$

Using (4.23) and standard estimates, we obtain

$$
\begin{aligned}
& E\left\{\left|\tilde{x}_{1}(r)-\tilde{x}_{0}(r)\right|^{4}\right\} \\
& \quad \leq K\left[\left(\left|y_{1}-y_{0}\right|^{2}+\left|s_{1}-s_{0}\right|^{2}\right)^{2}+\int_{s_{\lambda}}^{r} \mathbb{E}\left\{\left|\tilde{x}_{1}(\rho)-\tilde{x}_{0}(\rho)\right|^{4}\right\} d \rho\right] .
\end{aligned}
$$

Finally, thanks to Gronwall's Lemma, we deduce that

$$
\left(\mathbb{E}\left\{\left|\tilde{x}_{1}(r)-\tilde{x}_{0}(r)\right|^{4}\right\}\right)^{1 / 2} \leq K\left(\left|y_{1}-y_{0}\right|^{2}+\left|s_{1}-s_{0}\right|^{2}\right)
$$

which, in view of (4.30), gives the desired estimate (4.24).

Proof of (4.26). this result is a direct consequence of inequality (4.31) and the fact that $\tilde{x}_{i}(T)=x_{i}(T), i=0,1$.

Proof of (4.27). such an estimate can be deduced from the much more technical Lemma 4.3 given at the end of the present section.

Proof of (4.28). from the fact that $\tilde{x}_{i}$ and $x_{i}$ coincide at $T$, for $i=0,1$, we obtain

$$
\begin{aligned}
& \mathbb{E}\left\{\left|\tilde{X}_{\lambda}(T)-x_{\lambda}(T)\right|\right\} \leq \mathbb{E}\left\{\left|\tilde{X}_{\lambda}(T)-\tilde{x}_{\lambda}(T)\right|\right\}+\mathbb{E}\left\{\left|\tilde{x}_{\lambda}(T)-x_{\lambda}(T)\right|\right\} \\
& \quad \leq \mathbb{E}\left\{\left|\lambda \tilde{x}_{1}(T)+(1-\lambda) \tilde{x}_{0}(T)-\tilde{\lambda}_{1} \tilde{x}_{1}(T)-\tilde{\lambda}_{0} \tilde{x}_{0}(T)\right|\right\}+\mathbb{E}\left\{\left|\tilde{x}_{\lambda}(T)-x_{\lambda}(T)\right|\right\}
\end{aligned}
$$

Thus, by the definition of $\tilde{\lambda}_{i}, i=0,1$,

$$
\begin{aligned}
& \mathbb{E}\left\{\left|\tilde{X}_{\lambda}(T)-x_{\lambda}(T)\right|\right\} \\
& \quad \leq \lambda(1-\lambda) \frac{\left|s_{1}-s_{0}\right|}{T-s_{\lambda}} \mathbb{E}\left\{\left|\tilde{x}_{1}(T)-\tilde{x}_{0}(T)\right|\right\}+\mathbb{E}\left\{\left|\tilde{x}_{\lambda}(T)-x_{\lambda}(T)\right|\right\}
\end{aligned}
$$

Then, applying Hölder's inequality and taking into account (4.31) as well as (4.32) in Lemma 4.3 we infer that the latter estimate is dominated by

$$
K \lambda(1-\lambda)\left(\left|y_{1}-y_{0}\right|^{2}+\left|s_{1}-s_{0}\right|^{2}+\left|s_{1}-s_{0}\right|\left|y_{1}-y_{0}\right|\right) .
$$

This proves (4.28).

The above proof is completed by the following.

Lemma 4.3. Under our standard hypothesis we have

$$
\left(\mathbb{E}\left\{\left|\tilde{x}_{\lambda}(r)-x_{\lambda}(r)\right|^{2}\right\}\right)^{1 / 2} \leq K \lambda(1-\lambda)\left(\left|y_{1}-y_{0}\right|^{2}+\left|s_{1}-s_{0}\right|^{2}\right) .
$$


Proof of Lemma 4.3. Recalling the definition of $\tilde{x}_{\lambda}, \tilde{\lambda}_{1}$ and $\tilde{\lambda}_{0}$ we have

$$
\begin{aligned}
& \tilde{x}_{\lambda}(r)-x_{\lambda}(r)=\tilde{\lambda}_{1} \tilde{x}_{1}(r)+\tilde{\lambda}_{0} \tilde{x}_{0}(r)-x_{\lambda}(r) \\
& =\tilde{\lambda}_{1} y_{1}+\tilde{\lambda}_{0} y_{0}-y_{\lambda}+\int_{s_{\lambda}}^{r}\left[\lambda \dot{\tau}_{1}^{-2} b\left(\tau_{1}^{-1}(\rho), \tilde{x}_{1}(\rho), u_{\lambda}(\rho)\right)\right. \\
& \left.\quad+(1-\lambda) \dot{\tau}_{0}^{-2} b\left(\tau_{0}^{-1}(\rho), \tilde{x}_{0}(\rho), u_{\lambda}(\rho)\right)-b\left(\rho, x_{\lambda}(\rho), u_{\lambda}(\rho)\right)\right] d \rho \\
& \quad+\int_{s_{\lambda}}^{r}\left[\lambda \dot{\tau}_{1}^{-3 / 2} \sigma\left(\tau_{1}^{-1}(\rho), \tilde{x}_{1}(\rho), u_{\lambda}(\rho)\right)\right. \\
& \left.\quad+(1-\lambda) \dot{\tau}_{0}^{-3 / 2} \sigma\left(\tau_{0}^{-1}(\rho), \tilde{x}_{0}(\rho), u_{\lambda}(\rho)\right)-\sigma\left(\rho, x_{\lambda}(\rho), u_{\lambda}(\rho)\right)\right] d W(\rho)
\end{aligned}
$$

Taking the expectation of the square and using the Hölder as well as the Burkholder inequality we obtain

$$
\begin{aligned}
& \mathbb{E}\left\{\left|\tilde{x}_{\lambda}(r)-x_{\lambda}(r)\right|^{2}\right\} \leq K\left\{\left|\tilde{\lambda}_{1} y_{1}+\tilde{\lambda}_{0} y_{0}-y_{\lambda}\right|^{2}\right. \\
& +\mathbb{E}\left\{\int_{s_{\lambda}}^{r} \mid \lambda \dot{\tau}_{1}^{-2} b\left(\tau_{1}^{-1}(\rho), \tilde{x}_{1}(\rho), u_{\lambda}(\rho)\right)+(1-\lambda) \dot{\tau}_{0}^{-2} b\left(\tau_{0}^{-1}(\rho), \tilde{x}_{0}(\rho), u_{\lambda}(\rho)\right)\right. \\
& \quad-\left.b\left(\rho, x_{\lambda}(\rho), u_{\lambda}(\rho)\right)\right|^{2} d \rho+\mathbb{E}\left\{\int_{s_{\lambda}}^{r} \mid \lambda \dot{\tau}_{1}^{-3 / 2} \sigma\left(\tau_{1}^{-1}(\rho), \tilde{x}_{1}(\rho), u_{\lambda}(\rho)\right)\right. \\
& \left.\quad+(1-\lambda) \dot{\tau}_{0}^{-3 / 2} \sigma\left(\tau_{0}^{-1}(\rho), \tilde{x}_{0}(\rho), u_{\lambda}(\rho)\right)-\sigma\left(\rho, x_{\lambda}(\rho), u_{\lambda}(\rho)\right)\right\}\left.\right|^{2} d \rho
\end{aligned}
$$

In our next computations we will obtain suitable bounds for the terms in the right-hand side of the above inequality. This will be achieved by the following three estimates:

$$
\begin{aligned}
& \left|\tilde{\lambda}_{1} y_{1}+\tilde{\lambda}_{0} y_{0}-y_{\lambda}\right| \leq K \lambda(1-\lambda)\left|s_{1}-s_{0}\right|\left|y_{1}-y_{0}\right| \\
& \left(\mathbb { E } \left\{\mid \lambda \dot{\tau}_{1}^{-2} b\left(\tau_{1}^{-1}(\rho), \tilde{x}_{1}(\rho), u_{\lambda}(\rho)\right)+(1-\lambda) \dot{\tau}_{0}^{-2} b\left(\tau_{0}^{-1}(\rho), \tilde{x}_{0}(\rho), u_{\lambda}(\rho)\right)\right.\right. \\
& \left.\left.-\left.b\left(\rho, x_{\lambda}(\rho), u_{\lambda}(\rho)\right)\right|^{2}\right\}\right)^{1 / 2} \\
& \leq\left(\mathbb{E}\left\{\left|\tilde{x}_{\lambda}(r)-x_{\lambda}(r)\right|^{2}\right\}\right)^{1 / 2}+K \lambda(1-\lambda)\left(\left|s_{1}-s_{0}\right|^{2}+\left|y_{1}-y_{0}\right|^{2}\right) \\
& \left(\mathbb { E } \left\{\mid \lambda \dot{\tau}_{1}^{-3 / 2} \sigma\left(\tau_{1}^{-1}(\rho), \tilde{x}_{1}(\rho), u_{\lambda}(\rho)\right)\right.\right. \\
& \left.\left.+(1-\lambda) \dot{\tau}_{0}^{-3 / 2} \sigma\left(\tau_{0}^{-1}(\rho), \tilde{x}_{0}(\rho), u_{\lambda}(\rho)\right)-\left.\sigma\left(\rho, x_{\lambda}(\rho), u_{\lambda}(\rho)\right)\right|^{2}\right\}\right)^{1 / 2} \\
& \leq\left(\mathbb{E}\left\{\left|\tilde{x}_{\lambda}(r)-x_{\lambda}(r)\right|^{2}\right\}\right)^{1 / 2}+K \lambda(1-\lambda)\left(\left|s_{1}-s_{0}\right|^{2}+\left|y_{1}-y_{0}\right|^{2}\right)
\end{aligned}
$$

Proof of (4.34). This is obtained by the following straightforward computation $\left|\tilde{\lambda}_{1} y_{1}+\tilde{\lambda}_{0} y_{0}-y_{\lambda}\right|=\frac{\lambda(1-\lambda)}{T-s_{\lambda}}\left|s_{1}-s_{0}\right|\left|y_{1}-y_{0}\right| \leq K \lambda(1-\lambda)\left|s_{1}-s_{0}\right|\left|y_{1}-y_{0}\right|$.

Proof of (4.35). Hereafter, to simplify the notation, we will omit the dependence of functions on the independent variable: we will write, for instance, $x_{\lambda}$ 
instead of $x_{\lambda}(\rho)$. We also define $\Lambda=\lambda \dot{\tau}_{1}^{-2}+(1-\lambda) \dot{\tau}_{0}^{-2}, \hat{\lambda}_{1}=\lambda \dot{\tau}_{1}^{-2} / \Lambda$ and $\hat{\lambda}_{0}=(1-\lambda) \dot{\tau}_{0}^{-2} / \Lambda$, and we introduce the following notation

$$
\hat{x}_{\lambda}=\hat{\lambda}_{1} \tilde{x}_{1}+\hat{\lambda}_{0} \tilde{x}_{0}, \quad \hat{t}(\rho)=\hat{\lambda}_{1} \tau_{1}^{-1}(\rho)+\hat{\lambda}_{0} \tau_{0}^{-1}(\rho) .
$$

Then, by assumption (S3) we have, recalling (4.29) and (4.25),

$$
\begin{aligned}
\left|\lambda \dot{\tau}_{1}^{-2} b\left(\tau_{1}^{-1}, \tilde{x}_{1}, u_{\lambda}\right)+(1-\lambda) \dot{\tau}_{0}^{-2} b\left(\tau_{0}^{-1}, \tilde{x}_{0}, u_{\lambda}\right)-b\left(\rho, x_{\lambda}, u_{\lambda}\right)\right| \\
=\left|\Lambda \hat{\lambda}_{1} b\left(\tau_{1}^{-1}, \tilde{x}_{1}, u_{\lambda}\right)+\Lambda \hat{\lambda}_{0} b\left(\tau_{0}^{-1}, \hat{x}_{0}, u_{\lambda}\right)-b\left(\rho, x_{\lambda}, u_{\lambda}\right)\right| \\
\leq\left|\Lambda \hat{\lambda}_{1} b\left(\tau_{1}^{-1}, \tilde{x}_{1}, u_{\lambda}\right)+\Lambda \hat{\lambda}_{0} b\left(\tau_{0}^{-1}, \hat{x}_{0}, u_{\lambda}\right)-\Lambda b\left(\hat{t}, \hat{x}_{\lambda}, u_{\lambda}\right)\right| \\
\left.\quad+\left|(\Lambda-1) b\left(\hat{t}, \hat{x}_{\lambda}, u_{\lambda}\right)\right|\right\}+\left\{\left|b\left(\hat{t}, \hat{x}_{\lambda}, u_{\lambda}\right)-b\left(\tilde{t}, \tilde{x}_{\lambda}, u_{\lambda}\right)\right|\right. \\
\quad+\left|b\left(\tilde{t}, \tilde{x}_{\lambda}, u_{\lambda}\right)-b\left(\rho, x_{\lambda}, u_{\lambda}\right)\right| \\
\leq K \Lambda \hat{\lambda}_{1} \hat{\lambda}_{0}\left\{\left|\tau_{1}^{-1}-\tau_{0}^{-1}\right|^{2}+\left|\tilde{x}_{1}-\tilde{x}_{0}\right|^{2}\right\}+K\left|(\Lambda-1) b\left(\hat{t}, \hat{x}_{\lambda}, u_{\lambda}\right)\right| \\
\left.\quad+\left|b\left(\hat{t}, \hat{x}_{\lambda}, u_{\lambda}\right)-b\left(\tilde{t}, \tilde{x}_{\lambda}, u_{\lambda}\right)\right|+K\left\{|\tilde{t}-\rho|+\left|\tilde{x}_{\lambda}-x_{\lambda}\right|\right)\right\}
\end{aligned}
$$

Thus, from

$$
\begin{aligned}
& \hat{\lambda}_{1} \hat{\lambda}_{0}\left|\tau_{1}^{-1}-\tau_{0}^{-1}\right| \leq K \lambda(1-\lambda)\left|s_{1}-s_{0}\right|^{2} \\
& 0 \leq \Lambda-1 \leq K \lambda(1-\lambda)\left|s_{1}-s_{0}\right|^{2}, \quad|\tilde{t}-\rho| \leq K \lambda(1-\lambda)\left|s_{1}-s_{0}\right|^{2}
\end{aligned}
$$

and

$$
\begin{aligned}
|\hat{t}(\rho)-\tilde{t}(\rho)| & =\left|\left(\hat{\lambda}_{1}-\tilde{\lambda}_{1}\right) \tau_{1}^{-1}+\left(\hat{\lambda}_{0}-\tilde{\lambda}_{0}\right) \tau_{0}^{-1}\right| \\
& \leq \lambda(1-\lambda)\left|s_{1}-s_{0}\right|\left|\tau_{1}^{-1}-\tau_{0}^{-1}\right| \\
& \leq K \lambda(1-\lambda)\left|s_{1}-s_{0}\right|^{2}
\end{aligned}
$$

we get

$$
\begin{aligned}
\left\{\mathbb{E}\left|\lambda \dot{\tau}_{1}^{-2} b\left(\tau_{1}^{-1}, \tilde{x}_{1}, u_{\lambda}\right)+(1-\lambda) \dot{\tau}_{0}^{-2} b\left(\tau_{0}^{-1}, \tilde{x}_{0}, u_{\lambda}\right)-b\left(\rho, x_{\lambda}, u_{\lambda}\right)\right|^{2}\right\}^{1 / 2} \\
\leq K \lambda(1-\lambda)\left(\left|s_{1}-s_{0}\right|^{2}+K \hat{\lambda}_{1} \hat{\lambda}_{0}\left(\mathbb{E}\left\{\left|\tilde{x}_{1}-\tilde{x}_{0}\right|^{4}\right\}\right)^{1 / 2}\right. \\
\quad+K\left(\mathbb{E}\left\{\left|\tilde{x}_{\lambda}-x_{\lambda}\right|^{2}\right\}\right)^{1 / 2}+K\left(\mathbb{E}\left\{\left|\hat{x}_{\lambda}-\tilde{x}_{\lambda}\right|^{2}\right\}\right)^{1 / 2}
\end{aligned}
$$

In view of (4.31) we obtain

$$
\begin{aligned}
& \left(\mathbb{E}\left\{\left|\hat{x}_{\lambda}-\tilde{x}_{\lambda}\right|^{2}\right\}\right)^{1 / 2}=\left(\mathbb{E}\left\{\left|\left(\hat{\lambda}_{1}-\tilde{\lambda}_{1}\right) \tilde{x}_{1}+\left(\hat{\lambda}_{0}-\tilde{\lambda}_{0}\right) \tilde{x}_{0}\right|^{2}\right\}\right)^{1 / 2} \\
& \quad \leq \lambda(1-\lambda)\left|s_{1}-s_{0}\right|\left(\mathbb{E}\left\{\left|\tilde{x}_{1}-\tilde{x}_{0}\right|^{2}\right\}\right)^{1 / 2} \leq K \lambda(1-\lambda)\left|s_{1}-s_{0}\right|\left|y_{1}-y_{0}\right| .
\end{aligned}
$$

Consequently, using again (4.31)

$$
\begin{gathered}
\left\{\mathbb{E}\left|\lambda \dot{\tau}_{1}^{-2} b\left(\tau_{1}^{-1}, \tilde{x}_{1}, u_{\lambda}\right)+(1-\lambda) \dot{\tau}_{0}^{-2} b\left(\tau_{0}^{-1}, \tilde{x}_{0}, u_{\lambda}\right)-b\left(\rho, x_{\lambda}, u_{\lambda}\right)\right|^{2}\right\}^{1 / 2} \\
\leq K \lambda(1-\lambda)\left(\left|s_{1}-s_{0}\right|^{2}+\left|y_{1}-y_{0}\right|^{2}\right)+K\left(\mathbb{E}\left\{\left|\tilde{x}_{\lambda}-x_{\lambda}\right|^{2}\right\}\right)^{1 / 2}
\end{gathered}
$$

which in turn proves (4.35). 
Proof of (4.36). Let us define $\bar{\Lambda}=\lambda \dot{\tau}_{1}^{-3 / 2}+(1-\lambda) \dot{\tau}_{0}^{-3 / 2}, \bar{\lambda}_{1}=(\lambda / \bar{\Lambda}) \dot{\tau}_{1}^{-3 / 2}$ and $\bar{\lambda}_{0}=((1-\lambda) / \bar{\Lambda}) \dot{\tau}_{0}^{-3 / 2}$. Observe that $\bar{\lambda}_{0}+\bar{\lambda}_{1}=1$. One can easily check that $1-\bar{\Lambda} \leq K \lambda(1-\lambda)\left|s_{1}-s_{0}\right|^{2}$. Let us further introduce the notation

$$
\bar{x}_{\lambda} \doteq \bar{\lambda}_{1} \tilde{x}_{1}+\bar{\lambda}_{0} \tilde{x}_{0}, \quad \hat{t}(\rho) \doteq \bar{\lambda}_{1} \tau_{1}^{-1}(\rho)+\bar{\lambda}_{0} \tau_{0}^{-1}(\rho) .
$$

Then, we can rewrite the expression inside the expectation of the left-hand side of (4.36) as follows

$$
\begin{aligned}
&\left|\dot{\tau}_{1}^{-3 / 2} \sigma\left(\tau_{1}^{-1}, \tilde{x}_{1}, u_{\lambda}\right)+(1-\lambda) \dot{\tau}_{0}^{-3 / 2} \sigma\left(\tau_{0}^{-1}, \tilde{x}_{0}, u_{\lambda}\right)-\sigma\left(\rho, x_{\lambda}, u_{\lambda}\right)\right| \\
&=\mid\left|\bar{\Lambda} \bar{\lambda}_{1} \sigma\left(\tau_{1}^{-1}, \tilde{x}_{1}, u_{\lambda}\right)+\bar{\Lambda} \bar{\lambda}_{0} \sigma\left(\tau_{0}^{-1}, \tilde{x}_{0}, u_{\lambda}\right)-\sigma\left(\rho, x_{\lambda}, u_{\lambda}\right)\right| \\
& \leq\left|\bar{\Lambda}\left[\bar{\lambda}_{1} \sigma\left(\tau_{1}^{-1}, \tilde{x}_{1}, u_{\lambda}\right)+\bar{\lambda}_{0} \sigma\left(\tau_{0}^{-1}, \tilde{x}_{0}, u_{\lambda}\right)-\sigma\left(\hat{t}, \bar{x}_{\lambda}, u_{\lambda}\right)\right]\right| \\
& \quad+\left|(\bar{\Lambda}-1) \sigma\left(\hat{t}, \bar{x}_{\lambda}, u_{\lambda}\right)\right|+\left|\sigma\left(\hat{t}, \bar{x}_{\lambda}, u_{\lambda}\right)-\sigma\left(\tilde{t}, \tilde{x}_{\lambda}, u_{\lambda}\right)\right| \\
& \quad+\left|\sigma\left(\tilde{t}, \tilde{x}_{\lambda}, u_{\lambda}\right)-\sigma\left(\rho, x_{\lambda}, u_{\lambda}\right)\right| .
\end{aligned}
$$

From now on, we proceed in the same way as in the proof of (4.35), using first the regularity assumption (S3) and the boundedness of $\sigma$, and then equations (4.25) and (4.31). We can thus show that the previous expression is smaller or equal than

$$
\left\{\begin{array}{l}
\xi \doteq K\left[\bar{\Lambda} \bar{\lambda}_{1} \bar{\lambda}_{0}\left\{\left|\tau_{1}^{-1}-\tau_{0}^{-1}\right|^{2}+\left|\tilde{x}_{1}-\tilde{x}_{0}\right|^{2}\right\}+|\tilde{t}-\rho|+(\bar{\Lambda}-1)\right] \\
\quad+ \\
\eta \doteq K\left\{|\hat{t}-\tilde{t}|+\left|\bar{x}_{\lambda}-\tilde{x}_{\lambda}\right|+\left|\tilde{x}_{\lambda}-x_{\lambda}\right|\right\}
\end{array}\right.
$$

Using the same ideas as in the proof of (4.35), we obtain

$$
\left(\mathbb{E}\left[\xi^{2}\right]\right)^{1 / 2} \leq K \lambda(1-\lambda)\left(\left|s_{1}-s_{0}\right|^{2}+\left|y_{1}-y_{0}\right|^{2}\right)
$$

while

$\eta=\left|\left(\bar{\lambda}_{1}-\tilde{\lambda}_{1}\right) \tau_{1}^{-1}+\left(\bar{\lambda}_{0}-\tilde{\lambda}_{0}\right) \tau_{0}^{-1}\right|+\left|\left(\bar{\lambda}_{1}-\tilde{\lambda}_{1}\right) \tilde{x}_{1}+\left(\bar{\lambda}_{0}-\tilde{\lambda}_{0}\right) \tilde{x}_{0}\right|+\left|\tilde{x}_{\lambda}-x_{\lambda}\right|$.

Recalling the definition of $\bar{\lambda}_{i}, \tilde{\lambda}_{i}, \tilde{x}_{i}$ for $i=0,1$ a direct computation yields

$$
\eta \leq K\left[\lambda(1-\lambda)\left(\left|s_{0}-s_{1}\right|^{2}+\left|s_{0}-s_{1}\right|\left|\tilde{x}_{1}-\tilde{x}_{0}\right|\right)+\left|\tilde{x}_{\lambda}-x_{\lambda}\right|\right] .
$$

Consequently, from the estimates in the proof of (4.35)

$$
\begin{aligned}
& \left(\mathbb{E}\left\{\left|\dot{\tau}_{1}^{-3 / 2} \sigma\left(\tau_{1}^{-1}, \tilde{x}_{1}, u_{\lambda}\right)+(1-\lambda) \dot{\tau}_{0}^{-3 / 2} \sigma\left(\tau_{0}^{-1}, \tilde{x}_{0}, u_{\lambda}\right)-\sigma\left(\rho, x_{\lambda}, u_{\lambda}\right)\right|^{2}\right\}\right)^{1 / 2} \\
& \leq K\left[\lambda(1-\lambda)\left(\left|s_{0}-s_{1}\right|^{2}+\left|y_{0}-y_{1}\right|^{2}\right)+\left(\mathbb{E}\left\{\left|\tilde{x}_{\lambda}-x_{\lambda}\right|^{2}\right\}\right)^{1 / 2} .\right.
\end{aligned}
$$

This completes the proof of (4.36).

\section{References}

[1] Cannarsa, P., Sinestrari, C.: Semiconcave functions, Hamilton-Jacobi equations, and optimal control. In: Progress in Nonlinear Differential Equations and their Applications, vol. 58. Birkhauser, Boston (2004)

[2] Crandall, M.G., Ishii, H., Lions, P.L.: User's guide to viscosity solutions of Hamilton-Jacobi equations. Trans. Am. Math. Soc. 282, 452-502 (1984) 
[3] Fleming, W.H., Soner, H.M.: Controlled Markov processes and viscosity solutions. 2nd edn. Springer, New York (2006)

[4] Giga, Y., Goto, S., Ishii, H., Sato, M.-H.: Comparison principle and convexity preserving properties for singular degenerate parabolic equations on unbounded domains. Indiana Univ. Math. J. 40(2), 443-469 (1991)

[5] Ishii, H., Lions, P.L.: Viscosity solutions of fully nonlinear second order elliptic partial differential equations. J. Differ. Equ. 83, 26-78 (1990)

[6] Krylov, N.V.: Controlled diffusion processes. Applications of Mathematics. Vol. 14, Springer, New York-Berlin (1980)

[7] Wang, L.: On the regularity of fully nonlinear parabolic equations II. Comm. Pure Appl. Math. 45, 141-178 (1992)

[8] Yong, J., Zhou, X.Y.: Stochastic controls, Hamiltonian systems and HJB equations. Springer, New York (1999)

R. Buckdahn, M. Quincampoix

Laboratoire de Mathématiques, UMR6205,

Université de Bretagne Occidentale,

6 Avenue Le Gorgeu, 29200 Brest, France

e-mail: Marc.Quincampoix@univ-brest.fr

R. Buckdahn

e-mail: Rainer.Buckdahn@univ-brest.fr

P. Cannarsa

Dipartimento di Matematica,

Università di Roma Tor Vergata,

Via della Ricerca Scientifica 1,

00133 Roma, Italy

e-mail: cannarsa@mat.uniroma2.it

Received: 31 August 2009.

Accepted: 10 May 2010. 